\title{
Adopting the intentional stance toward natural and artificial agents
}

\author{
Jairo Perez-Osorio \& Agnieszka Wykowska \\ Istituto Italiano di Tecnologia (IIT) Genova, Italy
}

\begin{abstract}
In our daily lives, we need to predict and understand others' behaviour in order to navigate through our social environment. Predictions concerning other humans' behaviour usually refer to their mental states, such as beliefs or intentions. Such a predictive strategy is called adoption of the intentional stance. In this paper, we review literature related to the concept of intentional stance from the perspectives of philosophy, psychology, human development, culture and human-robot interaction. We propose that adopting the intentional stance might be a central factor in facilitating social attunement with artificial agents. The paper first reviews the theoretical considerations regarding the intentional stance, and examines literature related to the development of intentional stance across the life span. Subsequently, it discusses cultural norms as grounded in the intentional stance and finally, it focuses on the issue of adopting the intentional stance towards artificial agents, such as humanoid robots. At the dawn of the artificial intelligence era, the question of how (and when) we predict and explain robots' behaviour by referring to mental states is of high interest. The paper concludes with the discussion of the ethical consequences of robots towards which we adopt the intentional stance, and sketches future directions in research on this topic.
\end{abstract}

Keywords: intentional stance, social robotics, human-robot interaction, mental states.

\section{Introduction}

The ability to comprehend beliefs, desires or intentions of others is characteristic of, and necessary for, successful human interaction. It enables sharing mental representations, building common goals and acting in unison. Thanks to cognitive mechanisms specialised in perceiving social signals, humans and other primates are relatively good in anticipating others' behaviour, inferring mental states and taking others' perspective. The strategy of referring to others' mental states in order to predict their behaviour has been termed by the philosopher Daniel Dennett "adopting the intentional stance" (Dennett, 1987). This strategy is rapid and is adopted spontaneously. It generates predictions that adapt to various contexts, it is relatively efficient and accurate, and it works despite a lack of knowledge regarding the physical complexity underlying behaviour (for example, neuronal activity).

Facing the prospect of the introduction of robots into society, the question about social dynamics during interaction with robots emerge. It is plausible to think that the same social cognitive 
mechanisms that allow social attunement in human interactions might be activated during humanrobot interaction (HRI). We propose the term "social attunement" as an umbrella concept which encompasses all mechanisms of social cognition (e.g., mutual gaze, joint attention, or spatial perspective taking) activated during social interactions. Currently, robot designers have been focusing on providing robots with human-like appearance, behaviour, or communication skills in order to facilitate interaction. This has generated mixed results, as we will present in the following.

The present paper aims to review literature related to the concept of the intentional stance from the perspectives of philosophy, psychology, human development, culture and human-robot interaction. Such a review should provide the reader with a broad overview of the concept of intentional stance and the multifaceted approaches that have been undertaken to understand such mechanism. The paper first provides a summary of Dennett's idea of the intentional stance, then we review literature related to attribution of intentions and mental states from the developmental and cultural perspectives and then provides considerations regarding the foundations of the intentional stance and how this translates to human-robot interaction. The last part of the paper is dedicated to the discussion regarding the ethical implications of adopting the intentional stance toward humanoid robots.

\section{The three stances}

The brain has evolved to discriminate patterns and regularities in order to predict future states and events. From predicting a storm to understanding when others are bidding for attention, this "active perception" allows efficient functioning in complex environments. Humans use different strategies to understand and predict events. Dennett (1987, 1971) postulated three main strategies, dependent on the observed systems. For some systems, predictions are based primarily on the laws of physics and on the physical properties of things. Interactions between celestial bodies is an example. Such an approach is very accurate because it relies on the exhaustive understanding of most of the crucial variables that influence and interact with the system. When we make predictions depending on the physical properties of the system, we adopt the physical stance.

The main drawback of the physical stance is that it is impractical, as accessing the minutiae of some (complex) systems is very difficult or time-consuming. For those systems, predictions that refer to their functionality or design are more efficient. Think of a bicycle. It is not necessary to know how torque or gyroscopic precession keep the bicycle balanced to predict its behaviour. However, we know that if we keep on pedalling, it will stay in balance. In this case, when we generate predictions based on the design characteristics of the system we adopt the design stance. These predictions rely on additional assumptions or previous conventional knowledge regarding the purpose of the design. Once we know the purpose and functionality of an artefact, predictions become highly reliable and very practical because they circumvent the intricacies of the physical stance. The design stance can be applied to artefacts designed by humans or to living things designed by evolution. It can successfully describe the behaviour of animals, plants, organs or even a biome.

The behaviour of a particular class of complex living systems (humans) is best explained with reference to yet another stance, the intentional stance. The intentional stance is the strategy of interpreting the behaviour of an entity by treating it as a rational agent who makes behavioural 
choices in line with their desires and goals, and ways that $s /$ he believes will lead to the achievement of the set goals (Dennett, 2009). According to Dennett, for true intentional systems, intentional stance works best - it is the most efficient strategy to understand and predict their behaviour. Similar to the design stance, the intentional stance is a gamble that pays off in general. Thousands of years of evolution designed human beings to be rational, to believe what they ought to believe and want what they ought to want. The fact that we are products of a long and demanding evolutionary process guarantees that using the intentional strategy on ourselves is a safe bet. At its core, adopting the intentional stance is a bet for rationality. It implies that people's behaviour is ultimately regulated by their decisions and the capacity to execute them. For example, people exercise because: they want to be healthy, they desire to be in shape, and they believe that exercising will lead to being healthy and in shape.

Dennett borrowed the terms "belief" and "desire" from folk psychology, but he gives them a more technical meaning.

"My thesis will be that while a belief is a perfectly objective phenomenon [that apparently makes me a realist), it can be discerned only from the point of view of the one who adopts certain predictive strategy, and its existence can be confirmed only by an assessment of that strategy (that apparently makes me an interpretationist)"(Dennett, 1987, p.59).

Interestingly, he claims that the physical instantiation of these intentional states is irrelevant to the theory's predictive efficacy. Dennett also claims that any system whose behaviour can be best predicted by the intentional stance can be considered a true intentional system. Furthermore, he suggests that intentional stance is a craft, a practice of folk psychology, very useful to predict behaviour and used intuitively and automatically.

Although Dennett's perspective might fall short in explaining all the dimensions of the human behaviour (i.e., irrational actions), his postulates regarding how people use different predictive strategies to explain an agent behaviour are promising to understand human-robot interaction. In the case of social robotics, the question is whether/when and how people use mentalistic explanations to predict artificial agents' behaviour. Importantly, adopting the intentional stance towards an artificial agent does not imply that the agent has true intentionality. However, humans might treat agents as if they had mental states (Thellman et al. 2017). Therefore, adopting intentional stance does not require that artificial agents are endowed with mental states in the human sense, but rather that the user might be able to intuitively and reliably explain and predict their behaviour in these terms. Similarly to Dennett, rather than focusing on whether people actually believe that the agent has mental states, or whether people believe that an agent can have mental states at all, we focus on whether people explain and predict the behaviour of an artificial agent in mentalistic terms. This instrumentalist approach is similar to the Turing test, where instead of asking the question of whether a machine is intelligent, the question is whether and when people attribute intelligent behaviour to it.

It is crucial to differentiate the concept of intentional stance described by Dennett and the concept of Theory of Mind (ToM) used in cognitive science (Baron Cohen, 1995). Even though 
these two concepts are closely associated and can be subsumed under a common conceptual category, the context within which they have been introduced, and the way they have been empirically operationalised differ. As described above, the intentional stance is a strategy that allows prediction and explanation of the observed behaviour of a system. It can be tested comparing which strategy has been used to predict the system behaviour, physical, design or intentional stance. In contrast, the ToM is defined as the capacity of understanding others' specific mental states in a specific situation and use them predict and explain others behaviour in that given situation. This ability allows people to depart from their perspective and to comprehend others' misrepresented reality (false beliefs). The empirical test of the ToM compares different mental states (e.g., true vs false beliefs) whereas the test for intentional stance compares predictive strategies (i.e., physical vs design stance). Thus, ToM presupposes adopting the intentional stance, but not the other way around: it is possible to misinterpret others' mental states but still adopt the intentional stance towards them.

\section{Intentional stance and development}

Humans are very skilled at interpreting actions in terms of mental states. This skill seems to be acquired already at a very young age. However, children pass the explicit verbal false belief test (ToM test) only when they are four years old or older (Apperly, 2oII; Griffin \& Baron-Cohen, 2002; Wimmer \& Perner, 1983). However, in line with the logic proposed above, it might be that despite not having the ability to take the perspective of others, and thereby failing explicit ToM tests, younger children might still be capable of adopting the intentional stance. Therefore, multiple developmental studies have examined the cognitive mechanisms closely related to adopting the intentional stance before the robust appearance of the theory of mind (ToM) (Wimmer \& Perner, 1983). The present section briefly presents the most significant developmental findings regarding the understanding of others' actions by reference to mental states.

From a very early age, infants show an acute sensitivity to mental states underlying behaviour. For example, five-month-old infants have shown goal-attribution to objects that show animacy. In a habituation paradigm, infants looked longer at a hand reaching objects compared to a metallic rod (Woodward, 1998). At the same age, infants can follow the adult's gaze presented on the screen (Senju, \& Csibra, 2008) - which is deeply linked understanding of communicative-referential signals and to the appearance of affect attunement (sensitivity to others' emotions) (Stern, 1998). Recognition of gaze direction together with the recognition of animacy of movements, enable developing associations between agents' behaviours and its correspondent contingent effects in the environment, which leads to an early causal understanding of the world. Therefore, actions in familiar contexts begin to generate expectations. By nice months of age, infants understand that others have goals, that they are committed to achieving them and that different outcomes are linked to particular emotional states (i.e., success = happiness, or at least smiles) (Tomasello, Carpenter, Call, Behne, \& Moll, 2005). Around ten months, they parse streams of behaviour into units that correspond to what adults would see as separate actions (Baldwin, Baird, Saylor \& Clark, 200I).

At one year of age, crucial social behaviour emerges. Communicative gestures (i.e., pointing) and gaze following, both necessary for shared attention, become part of the children behavioural repertoire. Furthermore, detecting these social signals influences children's course of action 
(Sorce, Emde, Campos \& Klinnert, 1985; Feinman, \& Lewis, 1983). This suggests that the infant is coding the adult's mental state of attention to, or emotion about, a state of affairs. At this age, infants reliably follow the gaze of a faceless animal-like object if the object reacts contingently on their behaviour (Johnson, Slaughter \& Carey, 1998). Also, one-year-olds can differentiate whether the behaviour is goal-directed, based on the context in which behaviour unfolds. Fourteen- to eighteenmonth-old children will imitate a goal that has not been achieved by another person if the action is marked linguistically as purposeful, but not if it is marked as accidental (Carpenter, Akhtar, \& Tomasello, 1998). By I4 months of age, infants infer others' intentions and goals (Meltzoff, 1995). When they observed a person that fails to perform an action (trying to open the door while holding a big box) kids are more likely to help to complete the unsuccessful action rather than solely imitate the observed behaviour. From eighteen months, children can set aside their desires, recognise others' different desires and act accordingly (Repacholi \& Gopnik, 1997). When the experimenter asked to the kids "Can I have some food?" and showed interest in, for example, broccoli instead of biscuits, children gave the food preferred by the experimenter, rather than that preferred by themselves, while fourteen-months old children are more likely to give their own preferred food despite experimenter's preferences (Repacholi \& Gopnik, 1997). By fourteen months, infants start to understand intentional actions, meaning that behaviour unfolds following goal-oriented action plans and that attending to the goal-related elements of the action sequence is crucial for goal accomplishment (Tomasello, Carpenter, Call, Behne, \& Moll, 2005). Such understanding builds the foundation for imitative learning, a dominant form of cultural learning. Intention understanding not only allows predicting what others will do but also learning from them how to do things conventionally in their culture (Tomasello, Kruger, \& Ratner, 1993).

Two-year-old children appear to be capable of drawing pretended consequences from pretended assumptions. Harris (Harris, 1993) found that two-and-a-half-year-old children can distinguish between a pretend and a real outcome from a pretend or real assumption (e.g., that chocolate would be wet/dry after having pretended/real tea poured on it). They can differentiate pretended versus intended actions (e.g., Ma \& Lillard, 2006), also pretended versus intended but impossible (Behne, Carpenter, Call, \& Tomasello, 2005), and can identify individuals' preferences (e.g., Repacholi \& Gopnik, 1997). By three years of age, children start to understand the role of beliefs in intentional action and more accurately grasp the connections among desires, perceptions, and emotions (Tomasello, Carpenter, Call, Behne, \& Moll, 2005). Some researchers suggest that children around four years of age can predict behaviour based on a false belief (in a novel situation) (Wimmer \& Perner, 1983; Wellman, Cross, \& Watson, 20oI). Shortly after learning to speak, kids successfully use simple mental states terms like want, pretend, know, and think (Bartsch \& Wellman, 1995). However, 4- and 5-year-old children are unable to appreciate that, in order to pretend, an actor must have the intention to pretend (Lillard, 1998), and they struggle to distinguish between intentions and desires that relate to the same goal (Schult, 2002). Children's well-documented success in false-belief tasks at around 4 years of age (e.g., Wellman, Cross, \& Watson, 200I) is typically taken as evidence of their ability to represent mental states as such — an ability that applies to intentions just as it does for beliefs (Perner, 199I). However, children's conceptual understanding of intentions - of the motivational, causal, and epistemic components— begins before this age (Baird, \& Moses, 200I). 
Johnson (2003) examined the development of the representational system that serves the implementation of the theory of mind in children. In a series of experiments, they used stuffed animals with no person-like appearance and controlled them remotely. The experimenters manipulated two variables: presence or absence of facial features, and contingent or random behaviour of the object (i.e., led light or movement) with respect to children's behaviour. Findings suggest that I2- to I5-month-old infants interact with the object as if it had perceptual/attentional and communicative abilities and goal-directed behaviour. Based on these studies, the authors characterise five main cues that might trigger attribution of intentionality during infancy: (I) features like face and eyes; (2) an asymmetry along one axis, like having a head smaller than the body; (3) non-rigid transformations/movements such as expansion and contraction, contrary to linear changes; (4) self-propelled movement; and ( 5 ) the capacity for reciprocal and contingent behaviour. However, it remains unclear how the combination of these features and their interactions with the environment triggers attribution of intentionality, as not all asymmetrical or self-propelled agents evoke such attributions (Mar, \& Neil Macrae, 2008).

In summary, studies reveal that infants can use the context, the observed behaviour, and social signals to understand that others' actions are a result of mental states such as intentions. This skill appears by the end of the first year and gets robust around i8 months. It is clear that probably infants do not adopt the intentional stance in its fullest sense, as defined by Dennett (1987, 1997). Nonetheless, developmental findings agree that young children can understand 'simple mental states' such as desires, goals, intentions, attention, and perception (for a review see Johnson, 2003).

\section{Teleological stance}

Gergely and colleagues (e.g., 2003) suggest that those early acquired abilities are crucial for developing the full-fledged intentional stance adopted by older children and adults. Interestingly, these authors propose that at an early age, children only have a non-mentalistic interpretational system, the teleological stance. This system connects available information relevant to the task (i.e., actions, goal-states, and situational constraints) through the principle of rational action. Actions are oriented to achieve a goal state by the most efficient means available. Therefore, children can predict future states and have expectations regarding what is coming up next, without including others' beliefs and desires in the equation. Importantly, Gergely and Csibra suggest that the rationality principle implemented in the teleological stance is the same principle later applied in the mentalistic stance. This principle defines the general structure for action interpretation, as it presupposes that (I) actions aim to achieve future goal states, and (2) goal states are accomplished by the most rational action available to the agent within the constraints of the situation. The inferential structure creates the blueprint on which mental states can fit later. In particular, a mentalistic action explanation is acceptable if, and only if, the action (represented by the agent's intention) realises the goal state (represented by the agent's desire) rationally within the situational constraints (represented by the agent's beliefs). The elegant account suggested by Gergely and Csibra (2003) is more straightforward than the simulation theory approach, which suggests that in order to understand the purpose of the observed actions, infants would need to generate (simulate) others' mental states internally and subsequently attribute those mental states to the observed agent. Thus, the teleological stance theory 
suits more the cognitive abilities of pre-verbal children, who still lack the complex metarepresentational structures needed to represent intentional states (Leslie, 1987; 1994), and offers a framework for understanding the development of mental state attribution in infants.

Further support for the teleological stance comes from primate research. Several studies were designed to test whether primates are able to predict goal states of observed actions without having mental representations (for review Hauser \& Wood, 20I0). Authors developed a series of experiments in which a human throws an object towards an animal. Throwing is not part of primates' behavioural repertoire, meaning that they lack the internal representation of the throwing action. Therefore, predictions of the effects of throwing would be hard to explain with the simulation theory but can be explained using the teleological stance. Experimenters measured avoiding behaviour when the human threw an object to the animals, and manipulated different kinematic features of the movement (speed, angle, joints involved), the type of object/likelihood of harm (rock vs ball), and even whether the thrower was looking or not towards the animal. Stronger and more frequent avoiding behaviour was observed when the movement had full-fledged kinematics, the object was heavier and the attention was directed to the animal. Collectively, these findings suggest that predictions in primates are based to some extent on a teleological stance and that mental representations of actions (or simulation) are not required to anticipate action consequences.

The teleological stance theory implies that we can understand and predict others' behaviour through inferring action goals but without necessarily understanding their intentions. Evidence from animal and developmental studies suggest that this mechanism plays a role in action understanding. Observed movements and environmental constraints might be sufficient to explain certain behaviours without referring to mental states. Importantly, however, the inferential principle of the teleological stance can be the basis for mentalistic explanations, and reference to beliefs, desires, and intentions in later, verbal stages of development.

\section{Intentional stance and reproduction of cultural norms}

The skills crafted by infants during the first years of life set the foundations for social (and general) cognition. Interacting with others as intentional agents during this critical period helps children determining how others interpret their behaviour, how others respond to the behaviour and what it is expected from them in the future (Heider \& Simmel, 1944, reviewed by Malle, 20II). Furthermore, it enables multiple forms of cultural learning like imitation, instructional learning, use of tools and symbols, and acquisition of language (Searle, 1995). The development of these cultural tools contributes to the reproduction of the norms that structure human sociality because they are attained through the inter-individual agreement about the statuses of individuals, the entitlements and obligations they entail (Gilbert, 1990; Scholl, \& Tremoulet, 2000).

The transfer of human cultural tools from generation to generation relies on the intentional stance as the primary tool to understand and predict others' behaviour. Cognitive development at the early stages of life allows incorporation of societal norms into the children's behavioural repertoire. Adopting the intentional stance provides the flexibility to interact with others in variable and new social contexts. Importantly, as Michael (2015) describes, this process takes place because adults also adopt the intentional stance towards children. For instance, adults set up expectations regard- 
ing children's behaviour, in acquainting them with culture-specific elements, narratives, practices, social roles, among others. Adults - during the process of enculturalization of children - see children as potential rational, intentional agents, relying on the intentional stance as the principal strategy to predict behaviour. In this way, the reference to mental states is consolidated as the crucial foundation and force in social interaction. Additionally, it becomes a mechanism of cultural feedback (Michael, 20I5). Michael also argues that this feedback loop mechanism requires only that young children assume others to have mental states. Once this occurs, a common channel of communication is established between the infants and their caregivers, a channel mediated by mentalistic vocabulary (thanks to adopting the intentional stance). Furthermore, when infants follow others in adopting the intentional stance, they acquire better interpretational resources, which increases their incorporation into the adult environment, and this, in turn, furthers the process of enculturalization. This is how the intentional stance and cultural learning constitute a feedback loop (Michael, 2015).

Humans are raised immersed in the intentional stance. Extensive training and exposure to the intentional explanations start from very early childhood, and this makes humans experts in this way of explaining and predicting the behaviours of others. Therefore, the intentional stance becomes the most available explanatory/predictive strategy, even when other agents are not necessarily intentional systems. This results in a bias toward viewing agents as having goals, beliefs, and desires, and might provide an adaptive heuristic for understanding the world and the agents within. Despite the ease of adopting the intentional stance, humans are still able to identify true intentional systems. People understand that a computer is not tired, that a printer is not reluctant to work or that a volcano is not angry. Rather than assuming that such behaviour has been intentionally motivated (Searle, 1995), it is possible that humans interpret behaviours through the filter of "intentional states," as their most familiar, quick and effective way to predict behaviour. However, only if this strategy is the most efficient explanatory/predictive strategy, humans continue using it towards a specific agent. If another strategy explains a behaviour better, or if they are informed about a better/ most efficient strategy, they might change their stance. Intentional stance, however, might be distinctive in that adopting this strategy might trigger a more extensive set of social behaviours and social attunement, and might create an emotional connection with the observed agents. In summary, we propose that adopting intentional stance is a default, well-trained mechanism, selected by biological and cultural evolution, and a reliable tool in most social interactions with true intentional systems, allowing social attunement.

\section{Empirical evidence for adopting the intentional stance in adulthood}

Despite the relevance of abilities like mentalizing or attribution of intentionality in daily life, it has proven extremely difficult to evaluate them experimentally. It is clear that while people can evaluate the intentionality of behaviour on demand, they also spontaneously reason about mental states. Since the early approaches of Heider and Simmel (1944), researchers have attempted to understand the factors that trigger mentalizing. Heider \& Simmel (1944) presented a series of short animations, each involving two triangles (one large, one small) and a circle, all moving around an empty rectangle. Observers readily attributed personality traits to the shapes and described their movements 
in terms of mental states such as goals and emotions, a finding replicated by subsequent studies (for a review see Scholl \& Tremoulet, 200o). The pattern of the movements, rather than properties of the physical appearance of the interacting shapes (Scholl \& Tremoulet, 2000), triggers mentalistic descriptions (i.e., changes in the path, responding to other objects and self-propelled movement). This seminal study showed how readily people adopt the intentional stance even to abstract shapes. However, a question arises regarding the validity of the self-report method used to evaluate the spontaneous adoption of intentional stance. Critics suggest that the design of the experiment and the methods do not allow for ruling out the mediation of other higher-order cognitive mechanisms (Scholl \& Tremoulet, 200o). Participants' descriptions referring to perceived intentionality might be the result of higher-order cognitive mechanisms like inference from the questions or the task, rather than the actual observations.

In order to circumvent this caveat, more recently, researchers have chosen tasks with designs that do not require explicit judgement: neuroimaging approaches, complemented with questionnaires and semi-structured interviews. Some neuroimaging studies have explored the neural systems underlying mentalizing. This mechanism, closely related to adopting the intentional stance and attribution of intentionality, refers to reasoning about others' specific mental states (Frith \& Frith, 2000). Numerous tasks have been used to investigate mentalizing. Researchers used tasks such as observing and understanding the intentions and beliefs of characters in stories (Fletcher et al., I995; Gallagher, et al., 200o; Saxe \& Kanwisher, 2013) and cartoons (Brunet, Sarfati, Hardy-Baylé \& Decety, 2000; Fletcher et al., 1995, Vogeley, et al. (200I); reporting intentions — or lack thereof- during videos of animated geometrical shapes (Castelli, Happé, Frith, \& Frith, 200o) and predicting competitor's next action in a game (Fletcher et al., 1995). Independently of the task, these studies reported activation in the medial frontal cortex, the superior temporal sulcus (STS) especially around the temporoparietal junction (TPJ) and the temporal poles adjacent to the amygdala. All of these areas have been identified as part of the mentalizing network. Activity in the STS and TPJ have been also linked to detecting and understanding the intended biological motion (e.g., Allison, Puce, \& McCarthy, 2000; Pelphrey, Morris, \& McCarthy, 2004; Saxe et al., 2004). Moreover, activation in these regions seem to be triggered by the adoption of the intentional stance. Some studies revealed activation in these areas related to static images of features that cue intentionality, such as eyes, mouth, hands, and faces (for a review see Allison, Puce, \& McCarthy, 200o). Gallagher et al. (2002) observed activation in the medial anterior frontal cortex, related to the adoption of the intentional stance. The authors designed an experimental paradigm in which participants played a stone-paper-scissors game in the MRI scanner against agents that were believed to differ in terms of their intentional nature (a fellow human, an algorithm using specific rules, or a random number generator). Importantly, participants actually played against pre-programmed sequences in all three conditions. It was participants' beliefs about intentionality that triggered specific activation of the brain region aforementioned.

Empirical evidence for attribution of intentionality has been, however, a challenge. This is in part due to the difficulty of examining the emergence of attribution of intentionality using selfreport measures. Although new objective methods have shown neural correlates of adoption of the intentional stance and other structures involved in mentalizing, such as STS and TPJ, which are ac- 
tively involved in the general process of adopting the intentional stance, it is still not clear what process precisely we are examining when we address the issue of adopting the intentional stance.

\section{Intentional stance in social interaction with robots}

In the context of the new societal era, in which robots might soon share our environments, it is crucial to ask whether humans are likely to adopt the intentional stance towards embodied artificial agents, such as robots. In particular, humanoid robots, as those have some human-like characteristics of appearance. One could speculate that humans would not adopt the intentional stance towards a human-made artefact. In fact, this was confirmed by several findings: a study using a manipulation of the Prisoner's Dilemma (Krach et al., 2008) showed that areas associated with adopting the intentional stance in the medial prefrontal and left temporoparietal junction were not activated in response to artificial agents, whether or not they were embodied with a human-like appearance. Similarly, Chaminade et al. (2002) found that the neural correlates of adopting the intentional stance were not observed in interactions with artificial agents during a relatively simple rock-paperscissors game. These findings suggest that robots do not naturally induce intentional stance in the human interacting partner.

On the other hand, humans are prone to attribute human-like characteristics to non-human agents. Oral tradition and records from earlier civilisations and cultures reveal the tendency to anthropomorphise events or agents that show apparent independent agency: animals, natural events like storms or volcanos, the sun and the stars. This predisposition seems to have remained until today. Research shows the ease with which people provide anthropomorphic descriptions of agents (Epley, Waytz, \& Cacioppo, 2007 for review). Human-like motivations, intentions, and mental states might be attributed to also electronic or mechanical devices, computers, or in general agents that give the impression of a certain level of independent agency. Psychologically, anthropomorphism is considered a default and automatic psychological process (see Mitchell, Thompson \& Miles, 1997) that is often employed towards systems whose behaviour we do not understand, as it is the most available and familiar explanatory mode (Epley, Waytz, \& Cacioppo, 2007; Waytz, Epley, \& Cacioppo, 20Iо; Wiese, Metta \& Wykowska, 2017).

Interestingly, studies showed that anthropomorphism relies on the same cognitive mechanisms that generate attribution of the intentions to human behaviour (Castelli, Happé, Frith, \& Frith, 2000; Iacoboni, et al., 2004). The level of attribution can involve an assumption of mind, such as conscious experience, metacognition, and intentions (Gray, Gray \& Wegner, 2007), emotional states, behavioural characteristics, or human-like forms in non-human agents and in inanimate objects (Heider \& Simmel, 1944; also reviewed in Epley, Waytz, \& Cacioppo, 2007). Thus, it is plausible to hypothesise that humans might also adopt the intentional stance during the interaction with robots.

In line with this idea, Gazzola et al. (2007), as well as Oberman et al. (2007), showed that observing goal-directed actions performed by robots, such as grasping a wine glass, evoked similar mirror neuron system activity compared to when observing those actions performed by other humans. Similarly, Wykowska et al. (2014) showed that observing a robotic agent performing grasping and pointing actions biases perceptual processing in the same way as observing a human agent per- 
forming same actions. This indicates that people interpret robots, similarly to other humans, as goal-driven agents. More recently, Thellman, Silvervarg, and Ziemke (2017) presented a series of images and verbal descriptions of different behaviours exhibited either by a person or by a humanoid robot. Participants were asked to rate the intentionality, controllability, and desirability of the behaviours, and to judge the plausibility of seven different types of explanations derived from a recently proposed psychological model of lay causal explanation of human behaviour. Findings suggest that people adopted the intentional stance toward the robot to a similar degree as in the case of observing other humans.

Very recently, Marchesi et al. (2018) reported that people are prone to adopt the intentional stance towards humanoid robots, in specific contexts. The authors developed a questionnaire that explores the spontaneous adoption of intentional stance towards a humanoid robot iCub (Metta et al., 20IO). This instrument was created in order to evaluate whether people prefer to explain the behaviour of iCub using mentalistic or mechanistic terms. The questionnaire consists of 35 fictional scenarios depicting iCub performing different activities. In each scenario, a series of three pictures shows a sequence of events. Participants rate (by moving a slider on a scale) in each scenario if they think iCub's behaviour is motivated by a mechanical cause (referring to the design stance, such as malfunctioning, or calibration) or by a mentalistic reason (referring to the intentional stance, such as desire, or curiosity). The slider's scale has a mentalistic description on one extreme, and a mechanistic description on the other. First results with the use of this instrument showed that on average scores had a slight bias toward mechanistic explanations overall, which is not surprising, given that the depicted agent is a robot. This is in line with previous literature that suggests that people attribute a lower degree of intentionality to artificial agents' behaviour, compared to other humans (Krach, S. et al., 2008; Chaminade, T. et al., 2010; Wiese, Metta, and Wykowska, 2017). However, and interestingly, not all the choices in Marchesi et al.'s questionnaire were favouring mechanistic descriptions. Some items of the questionnaire scored predominantly mentalistic descriptions. Furthermore, also individual differences between participants were found, meaning that some participants were more likely to choose mentalistic explanations, while some other participants preferred mechanistic descriptions. Taken together, this suggests that factors such as the human-like appearance of the robot, the context in which the actions unfold, the apparent goal-oriented behaviour, as well as individual priors might affect the likelihood of adoption of the intentional stance towards artificial agents.

\section{Factors influencing the likelihood of adopting the intentional stance}

During social interactions, the brain uses highly sensitive mechanisms that are specialised to detect a broad spectrum of signals (i.e., facial expressions, changes in gaze direction). These social signals are in many cases taken as behavioural indicators of mental states. For example, when we see a frown, we infer that it indicates a person's disapproval. Therefore, the presence - or absence - of these indicators might be an essential factor in the likelihood of adopting the intentional stance. Hence, determining the specific parameters of those indicators that facilitate the adoption of the intentional stance is important and informative with respect to the design and implementation of behaviour in artificial agents. 
Research in human-robot interaction (HRI) shows that implementing human-like characteristics in artificial agents facilitates social interaction (see Fink, 2oI2; and, Fong, Nourbakhsh \& Dautenhahn, 2003, for review). Robots that show human-like appearance and behaviour are more accepted (Duffy, 2005; Goetz \& Kiesler, 2002; Venkatesh, \& Davis, 2000), more pleasant (Axelrod \& Hone, 2005), are perceived as more usable (Riek, Rabinowitch, Chakrabarti, \& Robinson, 2009), are easier to get acquainted with (Hegel et al., 2008), and are more engaging (Bartneck \& Forlizzi, 2004), relative to purely functional designs. Furthermore, robots that exhibit social signals like facial expressions (Eyssel, Hegel, Horstmann \& Wagner, 20I0), emotions (Gonsior et al., 20II) or turntaking in a conversation (Fussell, Kiesler \& Setlock, 2008) were more likeable and produced more emotional responses in participants. More recently, Willemse, Marchesi \& Wykowska (2018) showed, in a gaze-leading paradigm, that participants anthropomorphised and liked more those robots which followed the participants' gaze. In one condition of the experiment, a robot avatar looked $80 \%$ of the time at the same object that participants chose, whereas in another condition the robot was more likely to look at a different object (also 80\% of the time). Participants reported preferring the robot that followed their object preference and rated it as more human-like and as more likeable, relative to the one that did not follow the participant's gaze. Kompatsiari, Tikhanoff, Ciardo, Metta, \& Wykowska (2017) showed that mutual gaze established by a humanoid robot affected participants' judgments of human-likeness of the robot. Wykowska et al. (2015) showed that variability in temporal characteristics of gaze behaviour was perceived as human-controlled, despite that the behaviour was executed by a robotic agent (the iCub robot, Metta et al., 2010).

In summary, it seems that people might use various behavioural signals of an observed agent as hints that encourage adopting the intentional stance or anthropomorphising/ascribing humanness towards the agent.

\section{Consequences of adopting the intentional stance}

Understanding the conditions under which humans adopt the intentional stance towards artificial agents is not only of theoretical significance but might also have implications in terms of social interaction. Adopting the intentional stance towards an artificial agent might have multi-faceted consequences with respect to social attunement with the agent.

In line with the idea that adopting intentional stance might impact mechanisms of social cognition, Wiese, Wykowska, Zwickel \& Müller (2012) showed that people were more inclined to engage in joint attention with a robot when they believed its behaviour represented an intentional system. The authors used a gaze-cueing paradigm with robot and humans faces. In three experiments, they instructed participants differently regarding the agency of the observed behaviour. In the first experiment, without belief manipulation, human faces evoked a larger gaze-cueing effect compared to the robot faces. In the second and third experiment, participants were told that the observed gaze behaviour was the result of either mental operations (gaze behaviour controlled by a human) or was produced by an algorithm. Results showed a larger gaze cueing effects when participants believed that they were observing human-controlled behaviour (independent of whether they observed a human or a robot face), relative to the algorithm-generated behaviour. Further- 
more, Wykowska, Wiese, Prosser, \& Müller (2014) examined the electrophysiological correlates of this modulation of the gaze-cueing effect. Findings revealed larger amplitude of the PI component of the EEG signal (time window between IOo-I40 ms, locked to target presentation) for validly cued, versus invalidly cued targets when the behaviour was believed to be controlled by a human. This difference in amplitude was absent when participants believed the behaviour of the observed face was generated by an algorithm. Therefore, the PI effect mirrored behavioural findings observed in Wiese et al. (2012) and showed that the behavioural effects are due to early attention mechanisms. In sum, already early attention mechanisms are modulated by whether participants adopt an intentional or a design stance towards an agent.

These findings provide support to the notion that adopting intentional stance might influence the interpretation of basic social signals, and as a consequence, activation of fundamental mechanisms of social cognition.

\section{Different degrees of adopting the intentional stance}

As argued above, people seem to have a natural tendency to explain and describe others' behaviour in terms of mental states. This skill has been developed, extensively trained and proven useful in day-to-day interaction with other humans. We adopt the mentalistic stance spontaneously and effortlessly. However, it might be, that there are different degrees of intentional stance. At a more superficial level, the easiness of access to mentalistic explanations is reflected in the use of terms or expressions of the colloquial language: plants are sad, an engine refuses to start, the sea is angry. People do not actually believe that the sea has emotions. However, those simple and clear sentences are used as metaphors and convey the current state of affairs. It seems that mentalistic terms make communication efficient. The second level would include the attribution of human-like mental states to predict the behaviour of animals or other agents that do not necessarily have human-like mental representations. Interestingly, even though the behaviour of a dog or a cat could be possibly explained by the design stance (evolutionary design), people prefer to interact with them using the mentalistic stance. Attribution of mental states to the pet might work most of the time. Additionally, it creates a better rapport with the pet and might facilitate its inclusion into the social context. A third (or maybe second and a half) level would include understanding the behaviour of agents that pretend to have mental states as if they would have mental states. This refers to how the enjoyment of a theatre play, a novel or a cartoon strip relies on our engagement with the intentions and desires of the people involved. Spectators know the fictitious and predetermined nature of the characters. This makes movies or books entertaining, fun, emotive or meaningful. Although scripted and enacted, we willingly give life to characters using our skills of mental attribution, as we recognise that those represent the beliefs or desires of an actual intentional agent. Finally, at the most elaborated level, intentional stance unfolds as full-fledged mentalistic explanations of human behaviour based on others' beliefs and desires. The intentional stance, on which, for instance, a pedestrian aiming to cross the street would infer that drivers that see the red light will stop, as they do not have the intention to cause any harm to the pedestrians or other people. Interestingly, such reasoning can happen very quickly, perhaps even implicitly, while thinking about what to have for lunch or talking on the phone. This readiness for adopting the intentional stance and the capacity of ef- 
fortlessly switching between levels might be a crucial skill to navigate the complexity of the social environment. In summary, we hypothesise that people modulate the degrees of intentional stance in order to adapt to different social contexts in the most efficient way. Intentional stance might not be as precise in terms of explanations as physical or design stance, but sometimes one might invest less time and cognitive resources reducing precision but gaining fast, albeit rough, predictions. $\mathrm{Al}$ though the mentalistic strategy requires high-level cognitive skills, it can be easily accessible, and is also extremely flexible and often reliable.

Interestingly, the contexts in which social skills unfold have changed in recent years. Video games, virtual agents, and soon (humanoid) robots are pushing the readiness to adopt the intentional stance to its limits. Interaction with artificial agents might not require per se the engagement of mentalistic explanations, as suggested by Chaminade et al. (2005). If the behaviour of the system is predictable and efficiently explained by the design stance, the intentional stance would be a suboptimal strategy. One could explain the behaviour of a vending machine in mentalistic terms, but it might require some effort, would not flow spontaneously and could generate inaccurate predictions. However, as in the case of a pet, adopting the intentional stance towards humanoid robots might prove beneficial to include robots into the society, and to facilitate effective communication with others. Human-like physical features like eyes and face, together with biological motion could potentially provide the basis for mentalistic explanations. Moreover, including social skills in the robot could facilitate adopting the intentional stance, which might, in turn, facilitate social interaction.

\section{Ethical considerations}

Adopting the intentional stance seems to be a crucial factor in social interactions. Therefore, it seems also to be indispensable for smooth and natural interaction with robots. If adopting the intentional stance indeed facilitates social attunement, then it might be beneficial to design robots and robot behaviours that evoke the adoption of intentional stance: robots equipped with well-designed social mechanisms that would be able to adapt to a wide variety of social contexts and individual needs, that provide a custom-made level of social engagement, without compromising functionality. Most probably the robots that will interact first with humans at a larger scale would be service and social assistive robots. Robots with semi- or utterly autonomous behaviour that would perform goal-oriented tasks for- and with humans in specific social contexts: house, office, hospitals. Ideally, a social robot that evokes intentional stance, rather than being merely a tablet with wheels, should be able to signal its future states, recognise the needs of the humans and respond adequately. For example, if a person does not seem interested in establishing a human-like interaction with the robot, the robot would detect this attitude and adapt its communicative style (e.g., a minimal degree of eye contact, limited use of language and less spontaneous behaviour). On the other hand, if a robot would detect more signals from the human that invite for more social contact, the robot could increase its level of socially engaging behaviours. Robots easier to communicate with might be also easier to train and personalise (Torresen, 2018).

Robots that are engaging that learn and understand human behaviour and that attune socially with humans evoking the intentional stance could be used in an extremely beneficial manner 
as assistants and supporters in daily activities. Already today, robots that use social signals have proven beneficial in robot-assisted training of social skills for children diagnosed with autism spectrum disorder (ASD). According to several studies (for a review see Cabibihan, Javed \& Ang, 2013), individuals with ASD open up to interaction with robots, with which they can train their social behaviours and skills, such as emotion recognition or joint attention. Most importantly, those skills seem to be transferred from human-robot interaction to interaction with other humans (Dautenhahn, 2007; Kajopoulos et al., 20I5; Wykowska et al., 2015). Similarly, several studies speak in favour of the positive effects of social robots as companions for the elderly (see Broekens, Heerink, \& Rosendal, 2009 for review). In sum, robots seem to improve older adults' mood, decrease their feeling of loneliness and improve the connections with others. Additionally, subjective reports reveal that users enjoy the presence of companion robots. Despite that most of the studies have been made in nursing homes and have been limited to the Eastern populations, evidence suggests that current social companion robots might improve the quality of life of the elderly (Broekens, Heerink, \& Rosendal, 2009). Robots seem to be effective thanks to the possibility of establishing an emotional connection with the users. In this context, it is plausible to think that having robots that would attune to the users might increase the positive impact on the healthcare, and produce pleasant and more engaging social interactions.

In the future, robots that evoke the intentional stance, and thereby create social attunement, could potentially aid people in making different tasks at home, perform rather dull and repetitive tasks in hospitals, aid with surveillance, be placed at information desks, provide guidance in museums, and even become social companions. It is, however, possible that the implementation of social skills that provide robots with socially contingent behaviour might produce undesirable effects. For instance, users could experience a secure emotional attachment to the robots. Humans are already attached to many artefacts and cyber-technologies: cell phones, computers, video games, among others. Technology has been taking advantage of human cognitive resources to make them engaged. This facilitates incorporation of new technologies in people's lives, but with the drawback of making those services and products almost indispensable. The reason why these technologies are so attractive is a matter of discussion. Either because of physical features of apps or cellphones, due to an unstoppable avalanche of reward-oriented notifications, or the easiness to know the most recent social events instantly, people have incorporated and accepted these technologies in their daily lives and refuse to give them up. This has produced behavioural addictions to the internet, video games and the new pathologies related to mobile phones (i.e. nomophobia, no mobile phobia; FOMO: Fear Of Missing Out - the fear of being without a cell phone, disconnected or off the Internet, for review see De-Sola Gutiérrez, Rodríguez de Fonseca, \& Rubio, 2016). Studies reveal that although these pathologies appear in the general population, persons with psychosocial risk, loneliness, depression, and low self-esteem are more vulnerable to the excessive use of cyber-technological devices (Elhai et al., 2017). Thus, it is plausible to think that personalised social robots could generate emotional attachment or other types of bonds that might not have been yet studied. It is unclear at this point whether this type of attachment would have negative or positive consequences for humans. Vivid descriptions of the potential effects of socially capable robots have been described earlier in books like “The Bicentennial Man” or "Do Androids Dream of Electric Sheep?" and more re- 
cently depicted in movies like "Ex-machina" or TV shows like "Westworld". Consequently, it is crucial to discuss and define what type of bonding we are expecting to create with social robots.

Another potential problem is whether social robots will take people's jobs. This has been a concern of the public in general since the beginning of the industrial revolution and now growing stronger with the increase of the capacity in automation, robotics and artificial intelligence. However, statistics so far show that the introduction of information technology and automation has created far more jobs than those which are lost (Economist, 2016). In this context, social robots could potentially take over repetitive and heavy-duty tasks, giving the opportunity to improve the quality of life of the general population by increasing the time available for leisure and allowing people to do fulfilling jobs. Furthermore, social robots could help in to cope with the deficit in the workforce in certain types of jobs. For instance, there is already a shortage of healthcare workers and caregivers for the elderly. The need of workers in this area will only grow, as the population prospects indicate that by 2050 the percentage of people older than 65 would increase up to $16.7 \%$ globally (and more than $30 \%$ in Europe and Japan) (He, Goodkind, \& Kowal, 2016). Therefore, assistive and companion robots could undoubtedly give a helping hand in these areas.

Unfortunately, like on other occasions where humans have to face a significant societal change, many fears arise. These fears are broadcasted virally often without a critical, rational approach. The topics of artificial intelligence and robotics of the future are all over the news and media. Often these reports bring about unrealistic science-fiction-like scenarios for a technology that is still far from being as advanced as it is depicted there (see "Robots pave the way for our sci-fi future now" by TechCrunch, 2016; or any mainstream news about Sophia the robot as the new face and voice of artificial intelligence). It is very likely that similarly to previously adopted technologies, the market will determine the type of social interactions that humans and robots will have. We suggest that researchers and engineers across various domains should be called upon to monitor and evaluate preventively the potential implications of the introduction of social robots into human daily environments and design adequate measures to prevent or minimise these possible negative consequences. We think that the societal implications of introducing social robots that are very well attuned to humans are a very delicate topic which requires being addressed with a serious and rigorous approach from philosophy, psychology, anthropology, engineering, and law. All of these disciplines should actively participate in policymaking and being involved in their implementation.

Overall, it is essential to focus on research, and public outreach of research, on the potential advantages that the new robotic technologies might bring to society. We propose that developing robots which evoke the adoption of the intentional stance could have a positive impact on people's lives. However, this avenue should take into consideration possible emotional, psychological, political and economic consequences. Therefore, an extensive and in-depth interdisciplinary debate with a rational and critical evaluation of the state-of-the-art technology and its implications is indispensable. This debate should also involve the public and should be made open and very transparent.

\section{Future directions}

Recently, it has been suggested that in order to examine the social attunement between humans and robots one needs to use the methods of experimental psychology and cognitive neuroscience in 
natural (but experimentally controlled) human-robot interaction protocols (Kompatsiari PérezOsorio, Metta \& Wykowska, 2or8; Schellen, Pérez-Osorio \& Wykowska, 20ı8; Wiese, Metta \& Wykowska, 20I7, see also: www.instanceproject.eu). This allows for targeting and isolating specific cognitive mechanisms of the human brain and designing robots that evoked those specific mechanisms. The proper experimental control allows for better reproducibility and generalizability of results. One of the challenges is to translate the well-established paradigms of experimental psychology into more naturalistic interaction scenarios (Kompatsiari et al., 2018). This is because standard experimental protocols often present to participants reduced (in naturalness) stimuli, such as schematic drawings of faces. This is done in order to maintain experimental control. However, having already established robust and replicable effects with the use of such stimuli, it is now possible to move towards more ecologically valid protocols while still trying to maintain experimental control. This is precisely the approach we suggest. Once the standard protocols of experimental psychology are successfully transferred into HRI studies, it will be feasible to understand whether, and under what conditions, the well-studied mechanisms of human social cognition are evoked in HRI. By the same token, it is crucial to investigate objective (and perhaps implicit) measures of when people adopt the intentional stance towards robots. So far, more explicit measures have been developed, based on self-report, but future studies should be dedicated to discovering neural (and behavioural) correlates of subjective reports which indicate a mentalistic or mechanistic mode of explaining the observed behaviour. If such markers are identified, it would be attainable to determine - with objective implicit measures - whether, during an interaction with a robot, a participant is in the intentional or design stance mode. It might be beneficial for the interaction to online adjust behaviour of the robot in order to elicit one or the other stance, dependent on the current needs (if a robot needs to be perceived as a social entity, it might modify behaviour to evoke adoption of intentional stance, or, if it needs to be perceived instrumentally as a tool for a given task, it might behave in a manner that evokes the adoption of the design stance). Such online adaptation of behaviour is of course still in the realm of fantasy, but the goal of identifying neural or behavioural correlates of adopting the intentional stance is within the research agenda for the near future.

\section{Conclusions}

In the present paper, we review literature related to the concept of intentional stance from philosophical, developmental and HRI perspectives. The intentional stance, as postulated by Dennett, is the strategy of interpreting the behaviour of agents based on their intentions, desires, and goals. This strategy is advantageous and practical to understand and predict others' behaviour. People seem to adopt the intentional stance intuitively and automatically, even from very early stages of development. Evidence suggests that young children use social signals, behaviour, and context to explain and predict the behaviours of others. These early acquired abilities have been proposed to be the precursors of the full-fledged mentalistic intentional stance adopted by older children and adults. Based on this, we argued that adopting the intentional stance is a default, well-trained mechanism, selected by biological and cultural evolution, and a reliable tool in most social interactions, allowing social attunement. In this context, we propose that this powerful strategy might facilitate interaction with artificial agents. That is, designing robots that evoke the adoption of the intention- 
al stance towards them might facilitate social attunement and their integration into society. Finally, we propose that, in the process of developing robots with social capabilities that attune well with humans and potentially evoke adoption of intentional stance, we should actively discuss societal impact of such technology, and address potentially rising issues with reason and appropriate scientific methods.

\section{Acknowledgements}

Work on this paper, and research agenda described in "Future directions" section of the paper have been supported by the European Research Council under the European Union's Horizon 2020 research and innovation program (grant awarded to AW, titled "InStance: Intentional Stance for Social Attunement”. Grant agreement No.: ERC-2016-StG-715058). This paper is an extended version of a book chapter that will appear in the anthology "Wording Robotics" edited by Jean-Paul Laumond, Emmanuelle Danblon and Céline Pieters.

\section{References}

Allison, T., Puce, A., \& McCarthy, G. (200o). Social perception from visual cues: Role of the STS region. Trends in Cognitive Sciences. https://doi.org/Io.IoI6/SI364-6613(oo)oI50I-I

Apperly, I. (2oiI). Mindreaders: The cognitive basis of "theory of mind." Mindreaders: The cognitive basis of "theory of mind.” New York, NY, US: Psychology Press.

Axelrod, L., \& Hone, K. (2005). E-motional advantage: performance and satisfaction gains with affective computing. Proceedings of ACM CHI 2005 Conference on Human Factors in Computing Systems. https://doi.org/ IO.II45/1056808.1056874

Baird, J. A., \& Moses, L. J. (20oI). Do Preschoolers Appreciate That Identical Actions May Be Motivated by Different Intentions? Journal of Cognition and Development. https://doi.org/ro.I207/Sis327647JCDo204_4

Baldwin, D. A., Baird, J. A., Saylor, M. M., \& Clark, M. A. (20or). Infants parse dynamic action. Child Development. https://doi.org/Io.IIII/I467-8624.00310

Baron Cohen, S. (1995). Mindblindness. Learning, development, and conceptual change.

Bartsch, K., \& Wellman, H. M. (1995). Children talk about the mind. Children talk about the mind. New York, NY, US: Oxford University Press.

Behne, T., Carpenter, M., Call, J., \& Tomasello, M. (2005). Unwilling versus unable: Infants' understanding of intentional action. Developmental Psychology. https://doi.org/10.1037/oor2-1649.4I.2.328

Broekens, J., Heerink, M., \& Rosendal, H. (2009). Assistive social robots in elderly care: a review. Gerontechnology 2009; 8(2):94-I03

Brunet, E., Sarfati, Y., Hardy-Baylé, M. C., \& Decety, J. (2000). A PET investigation of the attribution of intentions with a nonverbal task. NeuroImage. https://doi.org/Io.1006/nimg.1999.0525

Cabibihan, J. J., Javed, H., Ang, M., \& Aljunied, S. M. (2013). Why Robots? A Survey on the Roles and Benefits of Social Robots in the Therapy of Children with Autism. International Journal of Social Robotics. https://doi.org/ I0.1007/s12369-013-0202-2 
Carpenter, M., Akhtar, N., \& Tomasello, M. (1998). Fourteen- through I8-month-old infants differentially imitate intentional and accidental actions. Infant Behavior and Development. https://doi.org/Io.Ioi6/ Sor63-6383(98)90009-I

Chaminade, T., Zecca, M., Blakemore, S.-J., Takanishi, A., Frith, C. D., Micera, S., ... Umiltà, M. A. (2oio). Brain Response to a Humanoid Robot in Areas Implicated in the Perception of Human Emotional Gestures. PLOS ONE, 5(7), eirs77. Retrieved from https://doi.org/ıo.I371/journal.pone.ooIr577

Dennett, D. (2009). Intentional Systems Theory. In The Oxford Handbook of Philosophy of Mind. https://doi.org/ Io.1093/oxfordhb/9780199262618.003.0020

Dennett, D. C. (1987). The Intentional Stance. MIT Press.

Dennett, D. C. (197I). Intentional systems. Journal of Philosophy, 68(February).

Dennett, D. C. (1997). True Believers: The Intentional Strategy and Why It Works. Mind Design. https://doi.org/ I0.1007/s13398-0I4-0173-7.2

De-Sola Gutiérrez, J., Rodríguez de Fonseca, F., \& Rubio, G. (2016). Cell-Phone Addiction: A Review. Frontiers in psychiatry, 7 , I75. doi:10.3389/fpsyt.2016.0oI75

Elhai, J., Dvorak, R., Levine, J. \& Hall, B. (2017) Problematic smartphone use: A conceptual overview and systematic review of relations with anxiety and depression psychopathology, Journal of Affective Disorders,Volume 207 (25I259) https://doi.org/ı.1016/j.jad.2016.08.030.

The Economist. (2016). Artificial intelligence: the impact on jobs - automation and anxiety. Economist. June 25th 2016. Available at: https://www.economist.com/news/special-report/21700758-will-smarter-machines-cause-mass-unemployment-automation-and-anxiety

Epley, N., Waytz, A., \& Cacioppo, J. T. (2007). On Seeing Human: A Three-Factor Theory of Anthropomorphism. Psychological Review, II4(4), 864-886. https://doi.org/Io.I037/o033-295X.II4-4.864

Feinman, S., \& Lewis, M. (1983). Social referencing at ten months: a second-order effect on infants' responses to strangers. Child Development. https://doi.org/Io.III/j.I467-8624.1983.tboo509.x

Fink, J. (20I2). Anthropomorphism and human likeness in the design of robots and human-robot interaction. Lecture Notes in Computer Science (Including Subseries Lecture Notes in Artificial Intelligence and Lecture Notes in Bioinformatics), 762I LNAI, 199-208. https://doi.org/10.1007/978-3-642-34103-8_20

Fletcher, P. C., Happé, F., Frith, U., Baker, S. C., Dolan, R. J., Frackowiak, R. S. J., \& Frith, C. D. (1995). Other minds in the brain: A functional imaging study of "theory of mind" in story comprehension. Cognition. https://doi.org/ I0.1016/0010-0277(95)00692-R

Fong, T., Nourbakhsh, I., \& Dautenhahn, K. (2003). A Survey of Socially Interactive Robots: Concepts, Design , and Applications Terrence Fong, Illah Nourbakhsh, and Kerstin Dautenhahn. Robotics and Autonomous Systems. https://doi.org/ı.IoI6/So92I-889o(o2)00372-X

Frith, C., \& Frith, U. (2000). The physiological basis of theory of mind: Functional neuroimaging studies. Understanding Other Minds: Perspectives from Developmental Cognitive Neuroscience.

Gallagher, H. L., Jack, A. I., Roepstorff, A., \& Frith, C. D. (2002). Imaging the intentional stance in a competitive game. NeuroImage. https://doi.org/ıo.Ioo6/nimg.2002.III7

Gazzola, V., Rizzolatti, G., Wicker, B., \& Keysers, C. (2007). The anthropomorphic brain: The mirror neuron system responds to human and robotic actions. NeuroImage. https://doi.org/ıo.Ior6/j.neuroimage.2007.02.003 
Gergely, G., \& Csibra, G. (2003). Teleological reasoning in infancy: The naïve theory of rational action. Trends in Cognitive Sciences. https://doi.org/ı0.1016/S1364-6613(03)0oI28-I

Gilbert, M. (1990). Walking Together: A Paradigmatic Social Phenomenon. Midwest Studies In Philosophy. https:// doi.org/IO.IIII/j.I475-4975.1990.tboo202.x

Goetz, J., \& Kiesler, S. (2002). Cooperation with a robotic assistant. In CHI 'O2 extended abstracts on Human factors in computing systems - CHI '02. https://doi.org/Io.II45/506443.506492

Gonsior, B., Sosnowski, S., Mayer, C., Blume, J., Radig, B., Wollherr, D., \& Kuhnlenz, K. (20II). Improving aspects of empathy and subjective performance for HRI through mirroring facial expressions. In Proceedings - IEEE International Workshop on Robot and Human Interactive Communication. https://doi.org/Io.IIo9/ ROMAN.2011.6005294

Gray, H. M., Gray, K., \& Wegner, D. M. (2007). Dimensions of Mind Perception. Science, 315(5812), 6r9 LP-6r9. Retrieved from http://science.sciencemag.org/content/315/5812/619.abstract

Griffin, R., \& Baron-Cohen, S. (2002). The intentional stance: developmental and neurocognitive perspectives. In Daniel Dennett, Brook, A., and Ross, D. (Eds) Cambridge, UK: Cambridge University Press, pp. 83-II6.

Hauser, M., \& Wood, J. (20I0). Evolving the Capacity to Understand Actions, Intentions, and Goals. SSRN. https:// doi.org/Io.II46/annurev.psych.093008.100434

Hegel, F., Krach, S., Kircher, T., Wrede, B., \& Sagerer, G. (2008). Understanding Social Robots: A User Study on Anthropomorphism. In Robot and Human Interactive Communication, 2008. RO-MAN 2008. The I7th IEEE International Symposium on. https://doi.org/Io.IIO9/ROMAN.2008.4600728

Iacoboni, M., Lieberman, M. D., Knowlton, B. J., Molnar-Szakacs, I., Moritz, M., Throop, C. J., \& Fiske, A. P. (2004). Watching social interactions produces dorsomedial prefrontal and medial parietal BOLD fMRI signal increases compared to a resting baseline. NeuroImage. https://doi.org/Io.IoI6/j.neuroimage.2003.II.oI3

Johnson, S. C. (2003). Detecting agents. Philosophical Transactions of the Royal Society B: Biological Sciences. https:// doi.org/ı.I098/rstb.2002.1237

Johnson, S., Slaughter, V., \& Carey, S. (1998). Whose gaze will infants follow? The elicitation of gaze following in I2month-olds. Developmental Science. https://doi.org/Io.1111/I467-7687.00036

Kajopoulos, J., Wong, A. H. Y., Yuen, A. W. C., Dung, T. A., Kee, T. Y., \& Wykowska, A. (20I5). Robot-Assisted Training of Joint Attention Skills in Children Diagnosed with Autism. In SOCIAL ROBOTICS (ICSR 2015). https:// doi.org/I0.I007/978-3-319-25554-5_30

Kompatsiari, K., Tikhanoff, V., Ciardo, F., Metta, G., \& Wykowska, A. (2017). The Importance of Mutual Gaze in Human-Robot Interaction BT - Social Robotics. In A. Kheddar, E. Yoshida, S. S. Ge, K. Suzuki, J.-J. Cabibihan, F. Eyssel, \& H. He (Eds.) (pp. 443-452). Cham: Springer International Publishing.

Kompatsiari, K., Pérez-Osorio, J., De Tommaso, D., Metta G. and Wykowska, A. "Neuroscientifically-Grounded Research for Improved Human-Robot Interaction," 2018 IEEE/RSJ International Conference on Intelligent Robots and Systems (IROS), Madrid, 2018, pp. 3403-3408.

Krach, S., Hegel, F., Wrede, B., Sagerer, G., Binkofski, F., \& Kircher, T. (20o8). Can machines think? Interaction and perspective taking with robots investigated via fMRI. PLoS ONE. https://doi.org/IO.I37I/journal.pone.0002597

Leslie, A. M. (1987). Pretense and Representation: The Origins of “Theory of Mind.” Psychological Review. https:// doi.org/I0.1037/0033-295X.94.4.4I2 
Leslie, A. M. (1994). Pretending and believing: issues in the theory of ToMM. Cognition. https://doi.org/Io.Ior6/ooro0277(94)90029-9

Ma, L., \& Lillard, A. S. (2006). Where is the real cheese? Young children's ability to discriminate between real and pretend acts. Child Development. https://doi.org/Io.IIII/j.I467-8624.2006.00972.x

Malle, B. F. (20II). Attribution Theories: How People Make Sense of Behavior. Theories in Social Psychology., 72-95.

Mar, R. A., \& Neil Macrae, C. (2008). Triggering the Intentional Stance. Empathy and Fairness, III-I2O. https:// doi.org/I0.1002/9780470030585.ch9

Meltzoff, A. N. (1995). Understanding the Intentions of Others: Re-Enactment of Intended Acts by I8-Month-Old Children. Developmental Psychology. https://doi.org/ıo.Io37/oor2-I649.3I.5.838

Metta, G., Sandini, G., Vernon, D., Natale, L., \& Nori, F. (2008). The iCub humanoid robot: an open platform for research in embodied cognition. In Performance Metrics for Intelligent Systems Workshop (PerMIS 2008).

Michael, J. (2015). The intentional stance and cultural learning: A developmental feedback loop. In Content and Consciousness Revisited. https://doi.org/IO.IOO7/978-3-319-I7374-0 9

Mullin, M. H., \& Mitchell, Robert W.; Thompson, Nicholas S.; Miles, H. L. (1997). Anthropomorphism, Anecdotes, and Animals. Current Anthropology.

Oberman, L. M., McCleery, J. P., Ramachandran, V. S., \& Pineda, J. A. (2007). EEG evidence for mirror neuron activity during the observation of human and robot actions: Toward an analysis of the human qualities of interactive robots. Neurocomputing. https://doi.org/Io.IoI6/j.neucom.2006.02.024

Pelphrey, K. A., Morris, J. P., \& McCarthy, G. (2004). Grasping the intentions of others: the perceived intentionality of an action influences activity in the superior temporal sulcus during social perception. Journal of cognitive neuroscience (Vol. 16). https://doi.org/Io.II62/o898929042947900

Perner, J. (199I). Understanding the Representational Mind. MIT Press.

Repacholi, B. M., \& Gopnik, A. (1997). Early reasoning about desires: evidence from I4- and I8-month-olds. Developmental Psychology. https://doi.org/Io.IO37/ooI2-I649.33.I.I2

Saxe, R., \& Kanwisher, N. (2013). People thinking about thinking people: The role of the temporo-parietal junction in “theory of mind.” In Social Neuroscience: Key Readings. https://doi.org/10.4324/9780203496190

Saxe, R., Xiao, D. K., Kovacs, G., Perrett, D. I., \& Kanwisher, N. (2004). A region of right posterior superior temporal sulcus responds to observed intentional actions. Neuropsychologia, 42(II), I435-I446. https://doi.org/IO.IOI6/ j.neuropsychologia.2004.04.015

Scholl, B. J., \& Tremoulet, P. D. (2000). Perceptual causality and animacy. Trends in Cognitive Sciences, 4(8), $299-309$. https://doi.org/IO.IOI6/Si364-66I3(oo)oI506-o

Searle, J. R. (1995). Construction of Social Reality. The Free Press.

Senju, A., Csibra, G., \& Johnson, M. H. (2008). Understanding the referential nature of looking: infants' preference for object-directed gaze. Cognition, Io8(2), 303-319. https://doi.org/Io.IoI6/j.cognition.2008.02.009

Sorce, J. F., Emde, R. N., Campos, J., \& Klinnert, M. D. (1985). Maternal Emotional Signaling. Its Effect on the Visual Cliff Behavior of I-Year-Olds. Developmental Psychology. https://doi.org/IO.IO37/oor2-I649.2I.I.I95

Stern, D. N. (1998). The Interpersonal World of the Infant: A View from Psychoanalysis and Developmental Psychology. New York Basic. https://doi.org/ıo.1017/CBO9781107415324.004 
Thellman, S., Silvervarg, A., \& Ziemke, T. (2017). Folk-psychological interpretation of human vs. humanoid robot behavior : Exploring the intentional stance toward robots. Frontiers in Psychology, 8(NOV), I-I4. https://doi.org/ I0.3389/fpsyg.2017.01962

Tomasello, M., Carpenter, M., Call, J., Behne, T., \& Moll, H. (2005). Understanding and sharing intentions: The origins of cultural cognition. Behavior al and Brain Sciences. https://doi.org/Io.IOI7/Sor40525Xo50oor29

Tomasello, M., Kruger, A. C., \& Ratner, H. H. (1993). Cultural learning. Behavioral and Brain Sciences. https:// doi.org/IO.IOI7/SoI40525X0003123X

Torresen J (2018) A Review of Future and Ethical Perspectives of Robotics and AI. Front. Robot. AI 4:75. doi: I0.3389/ frobt.2017.00075

Venkatesh, V., \& Davis, F. D. (200o). A Theoretical Extension of the Technology Acceptance Model: Four Longitudinal Field Studies. Management Science. https://doi.org/Io.I287/mnsc.46.2.186.11926

Vogeley, K., Bussfeld, P., Newen, A., Herrmann, S., Happé, F., Falkai, P., ... Zilles, K. (20or). Mind reading: Neural mechanisms of theory of mind and self-perspective. NeuroImage. https://doi.org/I0.1006/nimg.200I.0789

Wan, H., Goodkind, D., \& Kowal, P. (March 2016). "An Aging World: 2015 - International Population Reports" (PDF). United States Census Bureau. Retrieved 26 June 2016.

Waytz, A., Epley, N., \& Cacioppo, J. T. (2010). Social cognition unbound: Insights into anthropomorphism and dehumanization. Current Directions in Psychological Science. https://doi.org/IO.II77/0963721409359302

Wellman, H. M., Cross, D., \& Watson, J. (200I). Meta-analysis of theory-of-mind development: The truth about false belief. Child Development. https://doi.org/Io.IIII/I467-8624.00304

Wiese, E., Metta, G., \& Wykowska, A. (2017). Robots as intentional agents: Using neuroscientific methods to make robots appear more social. Frontiers in Psychology. https://doi.org/Io.3389/fpsyg.2017.01663

Wiese, E., Wykowska, A., Zwickel, J., \& Müller, H. J. (2012). I See What You Mean: How Attentional Selection Is Shaped by Ascribing Intentions to Others. PLoS ONE, 7(9). https://doi.org/I0.I37I/journal.pone.004539I

Willemse, C., Marchesi, S., \& Wykowska, A. (2018). Robot faces that follow gaze facilitate attentional engagement and increase their likeability. Frontiers in Psychology. https://doi.org/Io.3389/fpsyg.2018.00070

Wimmer, H., \& Perner, J. (1983). Beliefs about beliefs: Representation and constraining function of wrong beliefs in young children's understanding of deception. Cognition. https://doi.org/I0.IoI6/ooro-0277(83)90004-5

Woodward, A. L. (1998). Infants selectively encode the goal object of an actor's reach. Cognition. https://doi.org/ I0.IOI6/Sooro-0277(98)00058-4

Wykowska, A., Chellali, R., Al-Amin, M. Md., Müller, H.J. (2014). Implications of Robot Actions for Human Perception. How Do We Represent Actions of the Observed Robots? International Journal of Social Robotics, 6 (3), $357-366$.

Wykowska, A., Kajopoulos, J., Obando-Leitón, M., Chauhan, S. S., Cabibihan, J. J., \& Cheng, G. (2015). Humans are Well Tuned to Detecting Agents Among Non-agents: Examining the Sensitivity of Human Perception to Behavioural Characteristics of Intentional Systems. International Journal of Social Robotics. https://doi.org/ I0.1007/S12369-015-0299-6

Wykowska, A., Kajopoulos, J., Ramirez-Amaro, K., \& Cheng, G. (20I5). Autistic traits and sensitivity to human-like features of robot behaviour. Interaction Studies. https://doi.org/IO.IO75/is.I6.2.09wyk 